\title{
Implantação de um programa de mentoria remoto para estudantes de Medicina em tempo de pandemia
}

\author{
Implementation of a remote mentorship program for medical students during the pandemic \\ Sandra Regina Gonzaga Mazutti ${ }^{1}$ (D) sandra.mazutti@anhembi.br \\ Ana Cristina Kuhn Pletsch Roncati' ${ }^{1}$ ackpletsch@anhembi.br \\ Délio Eulálio Martins ${ }^{1}$ (D) deliomartins.br@gmail.com
}

\section{RESUMO}

Introdução: Programas de mentoria são necessários para apoio ao estudante de Medicina e podem ter diversos objetivos, dependendo das instituições que os implementam, embora ainda não sejam uma realidade na maioria das universidades. Durante a pandemia da Covid-19, encontros presenciais foram impedidos, o que dificultou ainda mais a manutenção desses programas, apesar de ser um dos momentos de maior necessidade emocional para os alunos. Ajustaram-se as aulas, e os encontros foram transformados em remotos, e, dessa forma, a mentoria on-line passou a ser uma realidade.

Relato de experiência: Apoiado pelo Núcleo de Apoio Psicopedagógico dos Estudantes de Medicina (Napem), foi estruturado um programa de mentoria em pequenos grupos, formados por docentes, discentes mais experientes (comentores) e mentorados (alunos do primeiro ao terceiro ano) com reuniões realizadas de forma remota, o que permitiu a participação dos estudantes, a interação com eles e o acolhimento desse público. As reuniões aconteceram entre junho e dezembro de 2020. O programa contou com a participação de 13 mentores, 94 alunos mentorados e 24 comentores. O programa teve uma sessão de treinamento com mentores e comentores, que foi conduzida pela psicóloga do Napem para alinhamento de expectativas e orientações. As discussões nos grupos focaram dificuldades na vida acadêmica, profissional e pessoal.

Discussão: O modelo remoto de mentoria apresenta como pontos fortes a maior flexibilidade para mentores e mentorados, a facilidade de participação de ambos e a manutenção da conexão do grupo, pois os encontros são facilitados pela tecnologia, visto que não houve nenhuma desistência. Como ponto fraco dessa experiência, citamos a falta de análises quantitativas e qualitativas do programa utilizando, por exemplo, entrevistas ou questionários de forma científica para avaliar melhor os resultados.

Conclusão: A mentoria remota é útil e deve ser incluída como uma possibilidade de legado permanente para os cursos de Medicina, pois os encontros mostraram-se relevantes para mentores e mentorados, e mantiveram-se com alta adesão durante todo o programa.

Palavras-chave: Mentores; Educação Médica; Tutoria; Pandemia.

\begin{abstract}
Introduction: Mentoring programs are necessary to support medical students and can have different objectives depending on the institution where they are implemented, although most universities have not yet reached that stage. During the Covid-19 pandemic, face-to-face meetings have been cancelled, making these programs even more difficult to maintain despite it being a time of even greater emotional need for the students. Classes were adjusted, and the meetings were transformed into remote sessions, making online mentoring a reality.

Experience report: Supported by the Center for Psychopedagogical Support of Students of Medicine (NAPEM), a mentoring program was structured in small groups, formed by teachers, more experienced students (co-mentors) and mentees (first to third year students) with meetings held remotely to enable participation, interaction and to welcome students. The program was attended by 13 mentors, 94 mentees and 24 co-mentors. The program included a training session with mentors and co-mentors, which was conducted by the NAPEM psychologist to align expectations and guidelines. Group discussions focused on difficulties in academic, professional and personal life.
\end{abstract}

Discussion: The advantages of the remote mentoring model are increased flexibility for mentors and mentees, facilitating their participation, and maintenance of the group connection supported by technology, since none of the mentees dropped out of the program. Weak points of this experience include the lack a quantitative and qualitative analysis of the program, for example through scientifically conducted interviews or questionnaires to produce better analysis of the results.

Conclusion: Remote mentoring is useful and should be included as a possible permanent legacy for medical courses, since the meetings were proven to be relevant to mentors and mentees and maintained a high rate of adherence throughout the program.

Keywords: Mentors; Medical Education; Mentoring; Pandemic.

${ }^{1}$ Universidade Anhembi Morumbi, São Paulo, São Paulo, Brasil.

Recebido em 01/03/21; Aceito em 07/04/21.

Editora: Patrícia Lacerda Bellodi

Avaliado pelo processo de double blind review. 


\section{INTRODUÇÃO}

A mentoria tem sido utilizada de forma mais ampla atualmente, mas foi implementada na educação médica a partir do final dos anos 1990'. Consiste em um programa de suporte e acompanhamento acadêmico que tem como objetivo auxiliar os alunos (mentorados) a alcançar determinados objetivos. Muitas definições podem ser utilizadas, mas o conceito de $\mathrm{Oxley}^{2}$ parece ser o mais abrangente quando define como um processo pelo qual uma pessoa experiente, altamente conceituada e empática (o mentor) orienta outro indivíduo (geralmente mais jovem) (o pupilo ou mentorado) no desenvolvimento e reexame de suas próprias ideias, sua aprendizagem e seu desenvolvimento pessoal e profissional.

Dessa forma, a mentoria é compreendida como um processo relacional, em que uma pessoa mais experiente utiliza a sua sabedoria e sensibilidade para acompanhar e orientar os iniciantes na sua trajetória de desenvolvimento pessoal e profissional ${ }^{3}$.

Os programas de mentoria podem ter diversos objetivos, que variam de acordo com as instituições, mas destacam-se as seguintes propostas: contribuir para a adaptação ao ambiente acadêmico, aumentar o interesse dos alunos, auxiliá-los no processo de desenvolvimento de habilidades, despertar o interesse pela medicina acadêmica e por outras áreas específicas, abordar questões relacionadas ao profissionalismo e superar o currículo oculto; potencializar a inserção de grupos minoritários na carreira médica, oferecer apoio emocional e aconselhamento, entre outras 4 .

No processo de mentoria, o relacionamento mentoraluno tende a ser mais informal, e deve ser incentivado o oferecimento de suporte em situações potencialmente estimulantes e desafiadoras, contribuindo para o desenvolvimento de profissionalismo e crescimento pessoal do aluno. Outro formato de mentoria bem conhecido é a mentoria entre pares que se apresenta como uma alternativa promissora de promover o desenvolvimento pessoal e profissional de alunos do primeiro ano do curso de Medicina 5 .

Durante o ano de 2020, a pandemia da coronavirus disease 2019 (Covid-19) modificou a vida de alunos e professores, e o isolamento social tornou-se uma preocupação ainda maior para a saúde mental dessa população. Além disso, a necessidade de mais conversas e compartilhamento de opiniões e sentimentos ficou ainda mais explícita.

Todos os alunos passaram a ter aula de forma remota, e os recém-ingressos não tiveram a oportunidade de conhecer pessoalmente os colegas ou professores e as facilidades e instalações do câmpus. Para os novos discentes, a mudança do ensino médio para a vida universitária ocorreu de forma ainda mais dramática e insegura. Esses alunos enfrentaram alta demanda de adaptação ao meio acadêmico, muitas vezes geográficas, com mudança de cidades e moradias, e principalmente relacionais entre pares, professores e outros profissionais.

Os alunos veteranos também vivenciaram dificuldades nunca vistas antes, o que gerou pânico, ansiedade, insegurança, medo, sensação de perda, entre outros sentimentos. Apesar disso, esses veteranos detinham uma experiência que poderiam compartilhar com outros alunos, criando um espaço de elaboração coletiva de questões vivenciadas no período de formação acadêmica e referentes à profissão médica.

Durante a pandemia, a adaptação ao mundo acadêmico tornou-se ainda mais difícil, e houve a necessidade de apoio para o desenvolvimento técnico e emocional dos discentes de forma humanizada. Nesse processo, um programa de mentoria para auxiliar nas dificuldades mais simples, detectar precocemente os confrontos mais significativos e providenciar encaminhamentos adequados, quando necessário, passou a ser ainda mais imperioso.

Dessa forma, diante do cenário atual de pandemia e isolamento social, em que encontros presenciais estavam proibidos, identificamos na mentoria on-line, em pequenos grupos, na forma mista entre pares e professores, uma abordagem distinta viável para implementação do programa de mentoria relacionada ao desenvolvimento dos estudantes, de modo a reafirmar o compromisso com a formação de profissionais íntegros, éticos e tecnicamente competentes.

\section{RELATO DE EXPERIÊNCIA}

O curso de Medicina da Universidade Anhembi Morumbi tem reconhecido, há vários anos, a importância de intervenções de suporte psicológico e pedagógico ao aluno: o Núcleo de Apoio Psicopedagógico dos Estudantes de Medicina (Napem) é exemplo dessa atenção, e a implantação do programa de mentoria ampliou a rede de suporte ao estudante.

O processo de implantação se deu em quatro fases:

1) Após aprovação do projeto pela coordenação do curso de Medicina, foi iniciada a divulgação do programa de mentoria para conhecimento de todos, e, posteriormente, enviou-se um convite aos professores e alunos do quarto ao sexto ano do curso de Medicina, que voluntariamente se candidataram para mentores e comentores (alunos), preenchendo um questionário por meio de um link emitido pelo Napem. No questionário, constavam perguntas de identificação do inscrito, no que acreditavam poder contribuir e quais as expectativas relativas à sua pretensão como mentor ou comentor. 
2) Devido ao período incomum e estressante para os alunos dos primeiros anos, consideramos interessante enviar um convite aos alunos do primeiro ao terceiro ano para que pudessem participar dos grupos de mentorados.

3) Os grupos foram formados de forma aleatória utilizando distribuição de um mentor, um ou dois comentores e em média sete alunos de primeiro ao terceiro ano como mentorados.

4) A psicóloga do Napem e a coordenadora do projeto realizaram uma reunião antes do início do programa para treinamento com professores mentores e alunos comentores, com a finalidade de alinhar expectativas e orientações sobre o objetivo da mentoria.

Como resultado das inscrições, tivemos 13 professores que voluntariamente se disponibilizaram preenchendo o questionário enviado, no qual expressaram a intenção de serem mentores. Foram realizadas 24 inscrições de alunos para a comentoria, sendo dez (42\%) do quarto ano, sete (29\%) do quinto e sete (29\%) do sexto.

Para participarem do grupo de mentoria, 94 alunos fizeram a inscrição, sendo 49 (52\%) do primeiro ano, 28 (30\%) do segundo e 17 (18\%) do ano.

As reuniões ocorreram de junho a dezembro de 2020, mantendo sempre os mesmos participantes. Realizaram, em média, 2,5 reuniões por grupo utilizando a plataforma virtual Microsoft Teams como ferramenta para os encontros remotos. Ao longo do semestre, por conta da sobrecarga causada pelas aulas e pelos estágios que deveriam ser recuperados pelo período de restrição devido à pandemia, nove alunos comentores desistiram do programa. Não houve nenhuma desistência de mentor ou mentorado.

Após cada reunião de mentoria realizada, os comentores enviaram à psicóloga do Napem um relatório no qual constavam o nome dos participantes, um resumo dos temas escolhidos e refletidos, e algumas frases expressas pelos mentorados com relação aos seus sentimentos vivenciados na reunião.

Nas conversas informais com a psicóloga, os mentores e comentores mencionaram a satisfação de estarem participando dos grupos e a importância do espaço de reflexão para que os alunos pudessem dispor de acolhimento e orientação sobre temáticas e vivências estressoras, e discutir acerca de problemas pessoais, relacionamento interpessoal e outros aspectos referentes ao curso, além de questões psicológicas na atuação prática cotidiana da formação médica que foram refletidas, avaliadas e cuidadas.
Os encontros, apesar de serem on-line, proporcionaram um bom vínculo entre professores e alunos, além de promoverem a troca entre os ingressantes e veteranos. Nesses encontros, os veteranos puderam contar as experiências prévias e fornecer informações sobre as alternativas de moradia, as melhores formas de estudo em disciplinas específicas e as escolhas que fizeram quanto às ligas acadêmicas.

Os temas se repetiram nos grupos, e os mais discutidos entre os alunos do primeiro ano foram os seguintes: a ansiedade pela expectativa da nova fase de vida e a adaptação como universitários; a frustração de não terem aulas presenciais, o que os impediu de conhecer os colegas de classe, os professores e os recursos oferecidos pela universidade, como os laboratórios de simulação; e dúvidas relativas às escolhas que fariam sobre diversas ligas existentes, diretório acadêmico e atlética.

Com relação aos alunos do segundo e do terceiro ano, os temas mais discutidos foram sobre o internato e a residência médica. No entanto, entre todos os alunos, os temas de maior interesse foram sobre a pandemia e as repercussões dela no desempenho acadêmico pelas aulas remotas, formas eficazes de estudos e gestão do tempo pela sobrecarga de conteúdo e atividades da rotina diária demandadas por seus pais, uma vez que retornaram para suas cidades de origem. Verbalizaram dificuldades com o ensino remoto, tais como falta de concentração, apatia, preocupação com seu desempenho acadêmico e, quando puderam retornar aos estágios, suas angústias diante da possibilidade de serem contaminados pelo novo coronavírus e transmitirem a doença para seus familiares.

\section{DISCUSSÃO}

Os estudantes do curso de Medicina ingressam na faculdade repletos de expectativas, dúvidas e ansiedades, especialmente no ano de 2020, quando grande parte dos ingressantes não teve a oportunidade de conhecer presencialmente a instituição com os recursos oferecidos, seus colegas e professores devido à pandemia da Covid-19. Contudo, à medida que puderam ter contato por meio dos encontros com mentores e comentores, dúvidas, medos e angústias puderam ser expressos e minimizados.

Fatores estressores, como mudanças de cidades, distância da família, sobrecarga curricular, provas de alta demanda para estudos, falta de supervisão próxima, ausência de suporte emocional, entre outros, acometem estudantes recém-ingressos no curso de Medicina ${ }^{6-8}$. Os relatos dos participantes dos alunos do primeiro ano denotam que puderam ser auxiliados na busca de respostas para suas dúvidas pertinentes à entrada num ambiente desconhecido, embora muito desejado.

Durante a pandemia, procuramos ofertar uma forma 
remota de mentoria respeitando os protocolos de afastamento social e ainda assim oferecer uma resposta à preocupação relacionada ao desenvolvimento dos estudantes, e reafirmar o compromisso com a formação de profissionais íntegros, éticos e tecnicamente competentes.

Logo no início do programa, uma das dificuldades foi definir o tamanho dos grupos. Embora não exista consenso sobre o tamanho ideal, optamos por manter grupos pequenos em torno de seis a oito alunos por mentor, para permitir que o grupo se conhecesse mesmo que por meio digital e fosse possível o suporte pessoal em vez de discussão apenas sobre desafios profissionais ${ }^{9}$. Entendemos que um programa de mentoria remoto também precisa ser de acompanhamento longitudinal com integração de docentes e alunos de diferentes semestres do curso, conforme recomendado por outros trabalhos ${ }^{10}$. Foram adotadas estratégias que propiciaram aos alunos o reconhecimento de seus sentimentos, suas emoções, fragilidades e potencialidades, contemplando durante a formação aspectos técnico-afetivos.

A implantação do programa de mentoria teve início em junho de 2020 de forma remota durante o período da pandemia causada pelo novo coronavírus. Os encontros aconteceram num momento de muitas mudanças e incertezas, porém pôdese constatar que foi útil e propício para a integração dos alunos, que puderam contar com o auxílio dos mentores e também com a troca de experiências dos comentores.

A periodicidade dos encontros não foi regulada, e cada grupo seguiu a cronologia que melhor se adequava aos seus membros, mas foi possível manter uma média de 2,5 reuniões por grupo. Embora existam vantagens desses encontros permitidos de acordo com a livre demanda do grupo, há a desvantagem de não garantir que todos os mentorados alcancem suas necessidades e seus anseios, nem que seja algo uniforme entre os grupos. Um ponto de atenção referese ao fato de que esse programa precisa ser avaliado, seja por questionários, entrevistas, métodos qualitativos como escalas de empatia ou mesmo por avaliações clínicas objetivas estruturadas, pois trata-se de métodos que permitem uma melhor análise dos resultados do programa $^{10}$.

A literatura aponta uma barreira para o funcionamento adequado de um programa de mentoria: a baixa adesão e participação dos alunos. Isso gera frustração nos mentores, pois a maioria se sente sobrecarregada por lidar com o programa como uma tarefa adicional e demorada ${ }^{11}$. Além disso, poucos programas remuneram os mentores ${ }^{10,12}$. No entanto, no modelo que desenvolvemos, não tivemos desistência nem queixas formais sobre a tarefa. Acreditamos que o fato de os encontros ocorrerem em horários mais flexíveis e a utilização da tecnologia favoreceram a menor sobrecarga dos docentes e também facilitaram a participação de alunos, que muitas vezes podem se sentir inibidos em reuniões em grupo realizadas no modelo presencial.

\section{CONCLUSÃO}

O modelo de mentoria remoto foi uma alternativa atrativa implementada durante a pandemia que apresentou vantagens fortes que justificam a sua manutenção mesmo quando o período de isolamento social acabar. Os encontros remotos não diminuíram a qualidade e a participação dos integrantes dos grupos, sendo possível a troca de experiências que aliviaram ansiedade e angústias expressas por mentorados.

\section{CONTRIBUIÇÃO DOS AUTORES}

Sandra Regina Gonzaga Mazutti colaborou na estruturação do programa, na aplicação do modelo, na aquisição de dados e na redação do artigo. Ana Cristina Kuhn Pletsch Roncati colaborou na interpretação de dados e na redação do artigo. Délio Eulálio Martins colaborou na interpretação dos dados e na redação e revisão do artigo

\section{CONFLITO DE INTERESSES}

Declaramos não haver conflito de interesses.

\section{FINANCIAMENTO}

Declaramos não haver financiamento.

\section{REFERÊNCIAS}

1. Buddeberg-Fischer B, Herta K-D. Formal mentoring programmes for medical students and doctors - a review of the Medline literature. Med Teach. 2006;28:248-57. doi: 10.1080/01421590500313043.

2. Oxley J, Briefing: Mentoring for doctors is a vague concept, though there seem. BMJ. 1998;317:3-3. doi: 10.1136/bmj.317.7165.3.

3. Frei E, Stamm M, Buddeberg-Fischer B. Mentoring programs for medical students - a review of the PubMed literature 2000-2008. BMC Med Educ 2010;10:32. doi: 10.1186/1472-6920-10-32.

4. Nimmons D, Giny S, Rosenthal J. Medical student mentoring programs current insights. Adv Med Educ Pract. 2019;10:113-23. doi.org/10.2147/ AMEP.S154974 [acesso em 10 mar 2021]. Disponível em: https://www. dovepress.com/medical-student-mentoring-programs-current-insightspeer-reviewed-article-AMEP.

5. Akinla $O$, Hagan $P$, Atiomo W. A systematic review of the literature describing the outcomes of near-peer mentoring programs for first year medical students. BMC Med Educ. 2018;18:98. doi: 10.1186/s12909-0181195-1.

6. Gan R, Snell L. When the learning environment is suboptimal. Acad Med 2014;89:608-17 [acesso em 10 mar 2021]. Disponível em: http://journals. Iww.com/00001888-201404000-00027.

7. Benbassat J. Changes in wellbeing and professional values among medical undergraduate students: a narrative review of the literature. Adv Heal Sci Educ. 2014;19:597-610. doi :10.1007/s10459-014-9500-1.

8. Coulehan J, Williams PC. Conflicting professional values in medical education. Camb Q Healthc Ethics. 2003;12:7-20 [acesso em 10 mar 2021]. Disponível em: https://www.cambridge.org/core/product/identifier/S0963180103121032/ type/journal_article. 
9. Tan YS, Teo SWA, Pei Y, Sng JH, Yap HW, Toh YP, et al. A framework for mentoring of medical students: thematic analysis of mentoring programmes between 2000 and 2015. Adv Heal Sci Educ. 2018;23:671-97. doi: 10.1007/s10459-018-9821-6.

10. Skjevik EP, Boudreau JD, Ringberg U, Schei E, Stenfors T, Kvernenes M, et al. Group mentorship for undergraduate medical students - a systematic review. Perspect Med Educ. 2020;9:272-80. doi: 10.1007/s40037-02000610-3.
11. Gonçalves MCN, Bellodi PL. Mentors also need support: a study on their difficulties and resources in medical schools. Sao Paulo Med J. 2012;130:2528 [acesso em 10 mar 2021]. Disponível em: http://www.scielo.br/scielo. php?script=sci_arttext\&pid=S1516-31802012000400009\&lng=en\&tlng=en.

12. Farkas AH, Allenbaugh J, Bonifacino E, Turner R, Corbelli JA. Mentorship of US medical students: a systematic review. J Gen Intern Med. 2019;34:26029. doi: 10.1007/s11606-019-05256-4. 